\title{
Experimental Investigation of Impacting Flow between a Sub-Scale Twin-Rotor Configuration
}

\author{
Ali Mehrabi, Ali Reza Davari* \\ Department of Aerospace Engineering, Science and Research Branch, Islamic Azad University, Tehran, Iran \\ Received 12 November 2019; received in revised form14 January 2020; accepted 10 May 2020
}

DOI: https://doi.org/10.46604/ijeti.2020.4933

\begin{abstract}
The objective of this paper is to conduct the experiments for a sub-scale model of a twin-rotor helicopter's fountain flow in the ground effect based on the pressure and thrust measurements in the hover flight. Firstly, the flow pattern, which leads to form the fountain flow and tests' requirements, is summarized according to the non-overlapping rotors' flow field characteristics. Then, a multipurpose test stand design is introduced. After that, the fountain flow pressure and velocity graphs are obtained. Finally, the tuft technique is used to visualize the fountain flow below the model's body and on the ground. In addition, confirmation of the fountain flow existence hypothesis is verified by thrust measurements and its influence on the efficiency of the twin-rotor. The result indicates that in the twin-rotor configuration, ground effect improves the lift force due to the fact that the fountain flow is more than its value in the single-rotor configuration.
\end{abstract}

Keywords: hover flight, fountain flow, ground effect, twin-rotor configuration, pressure distribution

\section{Introduction}

A deeper understanding has now been achieved about the unsteady aerodynamics than that of the past decades which sheds new light on the issue of helicopter performance. For a helicopter flying near the ground, the direction of flow induced by the rotor changes from vertical downwash to radial outwash due to interactions with the ground. The value of rotor disk loading determines the strength of this induced flow [1]. The outwash starts from the outboard region of the blade tip and flows away from the helicopter.

Although the ground effects is a well-known issue in helicopter performance, a lot remained to be discovered about the aerodynamics of twin-rotor configurations at the proximity of the ground. The latter is primary of concern during actual flight and in wind tunnel or hovering tests. For heights less than one rotor diameter above the ground, the hovering performance is strongly affected by the ground effect [2].

The flow field around the twin-rotor configuration systems can be divided into five regions, based on the dominant phenomena in each region. There is a relatively quiescent flow region under the airframe which is similar to a fountain flow, even though no measurements have already been reported to confirm this hypothesis. In the literature, this region is known as the fountain flow and is formed below the intersection of the two rotors where the flow is upward.

During a twin-rotor configuration hovers with no overlap between the rotors, there is a clear distance between them, in the upper surface of the airframe, the downwash, and spanwise flows from the blade tip vortices merging at the centerline of the fuselage. The clear distance will also form an upward unsteady fountain flow (Fig. 1). This flow recirculates into the rotors and has a significant influence on aerodynamic performance of the rotors. In hovering, the upper fountain flow causes a vertical 
drag force on the fuselage, which is called download. The experiments made to measure the download on the wings of tilt rotors in helicopter mode showed that the amount of its reverse effect was equal to $10-15 \%$ of the total rotor thrust and reduces the vehicle payload in hover out of ground effect [3-5].

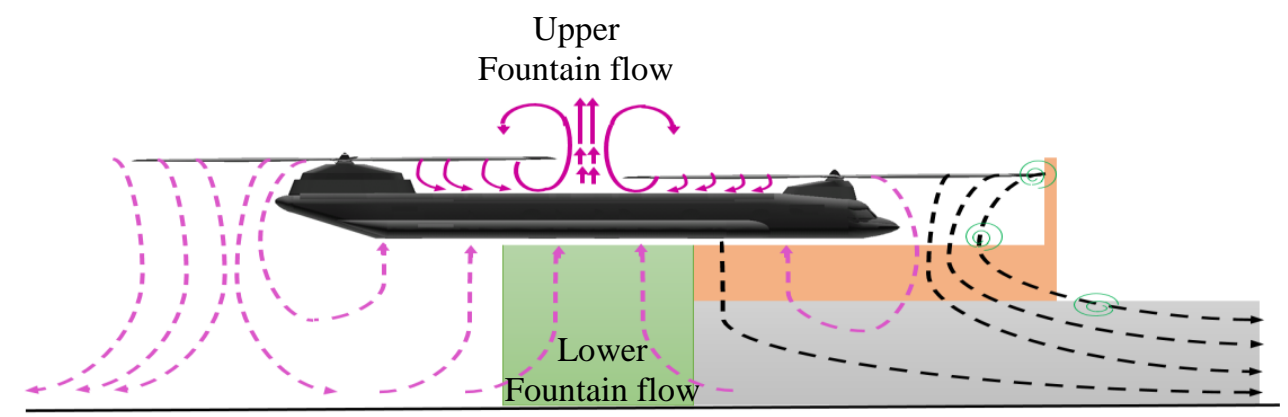

Fig. 1 Conceptual demonstration of the non-overlap tandem rotor fountain flow in ground effect

When a twin-rotor configuration hovers in the ground effect, the ground effect influences the wakes of the rotors under the fuselage (Fig. 1). A large part of the wakes recirculates as they interact with the ground surface and meet beneath the fuselage. It also forms a fountain flow in a similar form to what happens on the upper surface of the fuselage [6]. This fountain flow will be trapped under the surface of the fuselage and will cause an upward force on the twin-rotor configuration. The flow also reduces the download on the fuselage caused by upper surface fountain flow. Some measurements have showed that this positive upward force, could be as high as $9 \%$ of the total rotor thrust in tilt rotors in helicopter mode [7]. Therefore, due to the upper fountain flow, beneath the twin-rotor configuration body, the fountain flow increases the lifting capacity and payload beyond the maximum thrust of the rotors. Clearly, it is very important to know the effects of this flow on the performance of the twin-rotor configurations in different positions to make better performance in the hover mode. It seems that the flow pattern of the fountain flow in twin-rotor configurations varies with having overlap or without overlap. Investigating the effects of distance between twin-rotors and the ground effect role on the pressure and velocity values of the fountain flow below the generic twin-rotor configuration body is the main topic of the present study.

For a single rotor operating near the ground, the direction of the downwash flow in the vicinity of ground rapidly changes from vertical to radial along the surface, extending away from disk area. This region encompasses many turbulent vortices within the boundary layer with a wide range of length scales. When the rotor height above the ground decreases, it is observed that the vortex sheet is stretched with higher swirl velocities, resulting in less viscous diffusion and more pairing tip vortices [8]. This process preserves much longer vortex core original shape against the changes [9]. It is expected that the effects of this physical behavior of the flow will be observed under the airframe, which leads to the formation of a fountain flow. The pressure jump, tip vortices, and the vortex sheets across the blades impart remarkable oscillations in the velocity which must be taken into account in the fountain flow measurements.

Using the conservation of fluid momentum gives the rotor thrust (T), which can be obtained in [10]:

$$
T=\dot{m} \omega=\dot{m}\left(2 v_{i}\right)=2\left(\rho A v_{i}\right) v_{i}=2 \rho A v_{i}^{2}
$$

where $\omega$ and $v_{i}$ are the rotor downwash flow velocity and induced flow velocity respectively.

By non-dimensional analysis, the rotor thrust coefficient is defined in functional form in terms of density of air flow $\left(\rho_{\infty}\right)$, blade tip speed $\left(V_{t i p}\right)$, rotor disk area $(\mathrm{A})$, rotational velocity of the rotor $(\Omega)$, and rotor radius $(\mathrm{R})$ :

$$
C_{T}=\frac{T}{\rho_{\infty} A v_{t i p}{ }^{2}}=\frac{T}{\rho_{\infty} A \Omega^{2} R^{2}}
$$

equation (2) is based on the 1-D flow assumption, which means that the value of inflow is assumed to be distributed uniformly over the disk. 
For a rotor, the solidity represents the ratio of the lifting area of the blades $\left(A_{b}\right)$ to the area of the rotor $A$, which is:

$$
\sigma=\frac{A_{b}}{A}=\frac{N_{b} \bar{c} R}{\pi R^{2}}=\frac{N_{b} \bar{c}}{\pi R}
$$

The blade-loading coefficient can be expressed as:

$$
\frac{C_{T}}{\sigma}=\frac{T}{\rho A\left(\Omega R^{2}\right)}\left(\frac{A}{A_{b}}\right)=\frac{T}{\rho A_{b}\left(\Omega R^{2}\right)}
$$

Based on Cheeseman and Bennett's analysis, the ground effects on the rotor thrust can be expressed as:

$$
\left[\frac{T}{T_{\infty}}\right]_{P=\text { cons. }}=\frac{1}{1-\frac{\left(\frac{R}{4 z}\right)^{2}}{1+\left(\frac{\mu}{\lambda_{i}}\right)^{2}}}
$$

where $\mathrm{P}$ is the required power, $\mathrm{z}$ is the rotor height off the ground, $\lambda_{i}$ is the induced velocity at the rotor, and $\mu$ is the advance ratio in forward flight. For hovering flight and neglecting any blade-loading effects, equation (5) becomes:

$$
\left[\frac{T}{T_{\infty}}\right]_{P=\text { cons. }}=\frac{1}{1-\left(\frac{R}{4 z}\right)^{2}}
$$

According to these results, the effects of the ground on the rotor performance become significant for rotors hovering lower than twice rotor radius above the ground. So, the experiments in the present research are performed in rotors height $\mathrm{R}$ and $2 \mathrm{R}$ off the ground.

Several studies have already been devoted to the complicated flow field around the twin-rotor configurations and the role of ground effect on their performance. The results show that the aforementioned phenomena cannot be modeled by linear functions of independent variables such as thrust or height of rotors from the ground. Some of the recent studies have proposed empirical correction factors to estimate the flow field properties such as velocity. However, most of them are very case-dependent and can not be simply used for other cases. Moreover, there are still several unknown issues about the twin-rotor configurations which need to be discovered. For instance, in a tandem twin-rotor system, there is a strong interaction between the flow from the forward rotor and the aft rotor radial flow with airframe. Several experimental and numerical surveys are still needed to illuminate the existing understanding of the twin-rotor configurations performance.

The problem of twin-rotor configurations was first examined by Stepniewski [11]. He proposed an overlapping correction factor to improve the induced power estimation for twin-rotors. Several surveys have been devoted to analyze the performance of such rotors. Ramasamy [6] studied the downwash and outwash flow behavior by conducted experiments using a sub-scale $\mathrm{CH}-47 \mathrm{D}$ helicopter. He measured the flow-field velocities in a vertical plane at several azimuth angles and various radial distances from the rotor axis by using particle image velocimetry technique. He reported that there is a relatively quiescent flow region under the airframe which is likely to be similar to a fountain flow, but he didn't perform any measurement on that region.

Some studies on upper fountain flow of tilt-rotors in helicopter mode and side by side rotors were conducted to identify and reduce resultant downloads and their unfavorable consequences. These experiments were on major subjects like the effect of the tilt-rotor wing geometry on download value, the effect of the rotor location on fountain flow and download force, and the 
effect of parameters such as the ground effect on fountain flow of an actual tilt rotor configurations. It is important to note that in ground effects. Contrary to the mentioned configurations, the fountain flow-field of the tandem twin-rotors, even its non-overlap type, are more complex. Because of in the side-by-side arrangement of the tilt rotors, each rotor is less affected by the wake of the other one. However, in the tandem arrangement, each rotor, especially the rear rotor, is affected by vortices of the front rotor wake.

Experiments were reviewed to investigate the download force on upper surface of a tilt rotor-wing in Ref. [12]. In the mentioned research, the effects of the operational parameters such as wing flap size and wing flap deflection on the wing download were investigated. Fountain flow velocities of a full-span and a semi-span configurations with an image plane of 0.08-scale model of the XV-15 tilt-rotor were measured by hot-wire technique in Ref. [5]. It was observed that flow velocities have significant differences in upper fountain-flow region in comparison with other azimuth angles. It should be noted that the fountain flow is a free jet, so the flow behavior changes by the shear layer forming on a used image plane, and it is not actual.

In the recent years, with the advent of digital computers and numerical algorithms, valuable information has been obtained by using computational fluid dynamics [13]. However, accurate predictions at a reasonable computational time and costs are still the challenging issues [14]. Care must be taken in mesh generation and choice of turbulence model to properly capture the details of such complicated phenomena as fountain flow and the rotor wake structure [15]. Of course, in recent researches, computational fluid dynamics methods have been used. The comparison of them with experimental and actual results has shown the significant progress of these methods and their success in simulating and predicting aerodynamic details of fountain flow [16], but in contrary to the experimental results, there are still some offsets in the numerical simulation due to the blade tip vortices, downwash interference with the airframe, and the aerodynamic properties of the blades [13, 17].

Reviewed studies showed that fountain flow between the twin-rotor configurations is a new discovered phenomenon and no case has been reported on experimental investigations relating to that under the tandem twin-rotor configuration body. On the other hand, most of the experimental investigations have already reported, have been performed in wind tunnels or special hover chambers in which the flow blocked by the chamber or tunnel walls deteriorates the flow field and makes it different from flow conditions occurring in a real flight. In the simulation of free flow on the rotors, the effects of the tunnel walls can hardly be eliminated. Despite using various methods to remove the effects of the boundary layer on the wind tunnel or chamber walls, a majority of the discrepancies observed between the wall-confined experimental data and those obtained during actual tests are believed due to the wall effects [18]. The ground proximity effect is also a missing issue in most of the experimental investigations. Very limited works were devoted to a detailed study on the effects of twin-rotor elevation from the ground on the flow field, which necessitates a large test section wind tunnel to eliminate any wall interaction and blockage effects.

To avoid the remarkable costs of such tunnels and the consequent problems, such as its turbulence level, velocity limitations, and so on, a versatile test stand has been used in the present study to investigate the fountain flow characteristics below a subscale twin-rotor airframe in the absence of any wind stream, with less surrounding wall effects. Various hovering elevations can be examined both in ground effect and outside it. This test rig is capable of measuring the pressure and velocity distributions in hover. So, in this paper, the effects of a complete body and twin-rotor configuration without overlapping blades have been studied on the fountain flow behavior of a detailed sub-scale model at two different heights above the ground with no surrounding wall effects. The flow visualization under the model airframe were conducted by the tuft technique to investigate the flow pattern changes caused by impacting flow.

Tuft flow visualization techniques were used in some prior studies to investigate the characteristics of downwash and outwash of the model and full-scale single main rotor helicopters and tilt-rotors [3]. The results of the tuft based on the experiments on the Blackhawk UH-60 helicopter body were used to validate the results of the numerical Navier-Stokes computational method. In these experiments, during the helicopter operation in hover, the unsteady patterns formed in the tufts mounted on the ground showed the outwash. 


\section{Experimental Approach}

The overall test rig encompasses three subsystems: the main stand, the data-acquisition section, and data entry and record section shown in Fig. 2. Motor modules, the airframe, rotors, and pressure sensor tubes, are installed in the main stand. The main stand height can be changed vertically to vary the ground effect. The boxes of the pressure sensors and signal amplifiers are located in the data-acquisition section. Sixteen pieces of precise and sensitive differential pressure sensors with output recording rate of $22 \mathrm{~Hz}$ were employed to measure the total pressures incoming through ports. Therefore, 770 samples were recorded in 35 seconds by the data acquisition system during the testes. This sampling rate is enough to capture the periodic variations and unsteadiness in the flow field. The pressure range of the pressure sensors is -2.0 to $+2.0 \mathrm{kPa}$. Their response time and warm-up time are 1.0 and $20 \mathrm{~ms}$ respectively. Typical errors of the pressure sensors over $+10^{\circ} \mathrm{C}$ to $+60^{\circ} \mathrm{C}$ are $2.5 \%$, with auto-zero system, and $6.25 \%$ maximum error without auto zero. Auto-zero is defined as storing the zero pressure output reading and subtracting this from the device's output during normal operations.

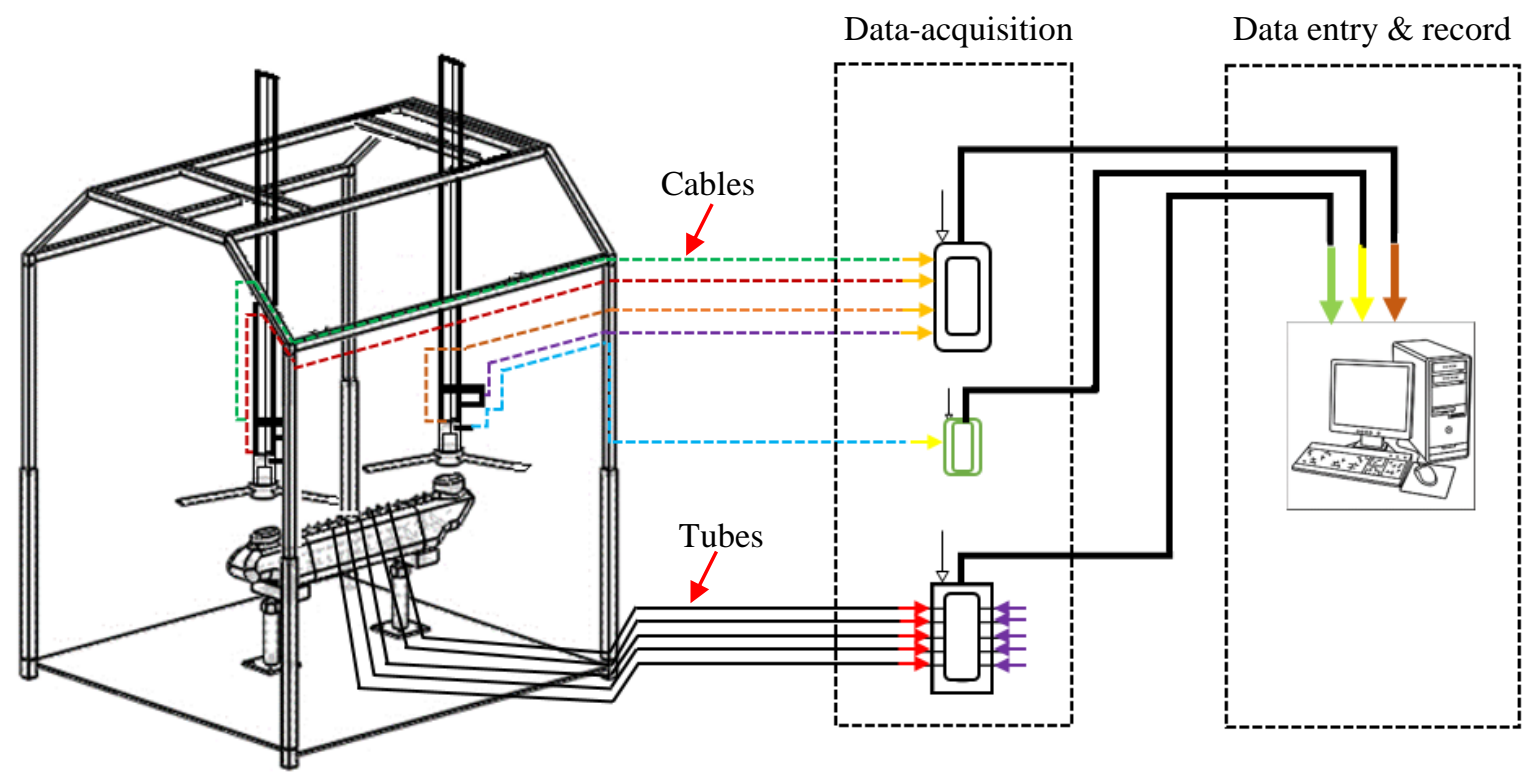

\begin{tabular}{|c|l|c|l|}
\hline Load cell output voltage & $\longrightarrow$ & Exciting voltage & $\longrightarrow$ \\
\hline Static pressure & $\longrightarrow$ & Load cell data & $\longrightarrow$ \\
\hline Total pressure & $\longrightarrow$ & Pressure data & $\longrightarrow$ \\
\hline Load cells board box & $\square$ & RPM. data & \\
\hline Pressure sensor's box & & RPM. Sensor box & \\
\hline
\end{tabular}

Fig. 2 Schematic of the test stand

In contrast to many of the previous researches, the present experiments have been performed in a big test environment where the wind tunnel or hover chamber walls, and the consequent flow blockage and effects are very low and negligible. Therefore, more reliable data have been achieved. The pressure ports are drilled along the longitudinal centre line of the fuselage to measure the pressure and velocity distribution below the body of the model. The ports have been located in specified distances from together. The ports consist of tiny steel probes of $2.5 \mathrm{~mm}$ external and $1.5 \mathrm{~mm}$ internal diameter. The probes were mounted by the way that they are fully tangent to the underside of the airframe. Each probe was directly connected to a differential pressure transducer via a plastic tube.

The purpose of the present study is to measure the pressure and velocity changes caused by impacting fountain flow at different points below the fuselage of a twin-rotor configuration and investigate the blades aerodynamic interaction on the fountain flow pattern by flow visualization. Two sets of constant pitch rotor blades were employed. A schematic view of the 
rotors arrangement and the longitudinal position of the associated pressure ports shown in Fig. 3. First set of the tests were performed using a single (forward) rotor, and then the second set of the tests done by tandem rotors. The experiments were carried out for two vertical distances from the ground; $h / 2 \mathrm{R}=0.5$ and $\mathrm{h} / 2 \mathrm{R}=1$. Both single-rotor and twin-rotor configuration were tested to compare the impacting fountain flow fields as well as the ground proximity effects.

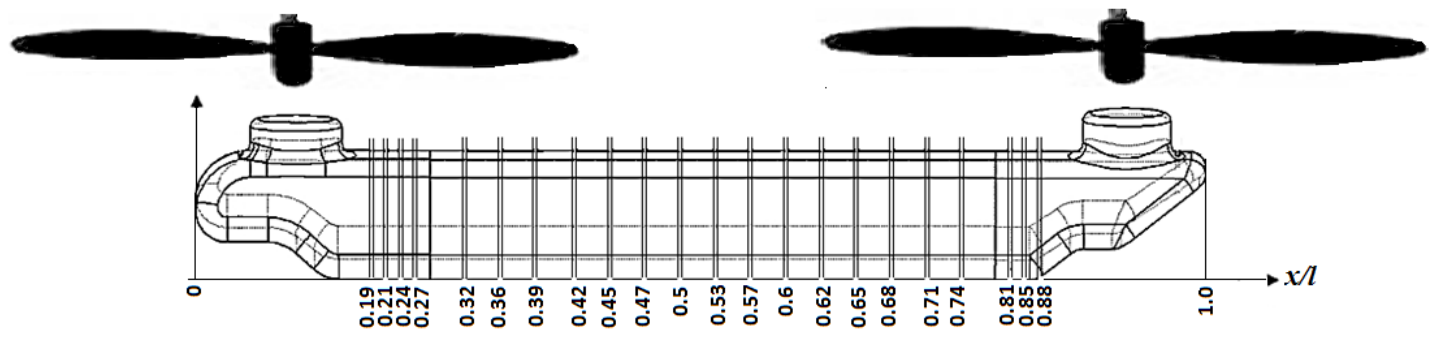

Fig. 3 The schematic view of twin-rotor configuration and embedded pressure ports

In order to physically understand the airflow patterns leading to the impacting fountain flow, special tuft flow visualization techniques are used in the present study. A series of white tufts planted at the black background were attached to the bottom surface of the body (Fig. 4). The tufts were $2.5 \mathrm{~cm}$ in length and were arranged in $3 \mathrm{~cm}$ square grids. Another white tuft flags were planted at the black plane on the ground to complete flow capturing below the rotors (Fig. 5). The tufts in this plane were $4.5 \mathrm{~cm}$ in length and were mounted on very thin wooden rods at height of $5 \mathrm{~cm}$ as flag. They were arranged in $10 \mathrm{~cm}$ square grids. The tufts were extremely light and flexible, so they would move freely with the flow field.

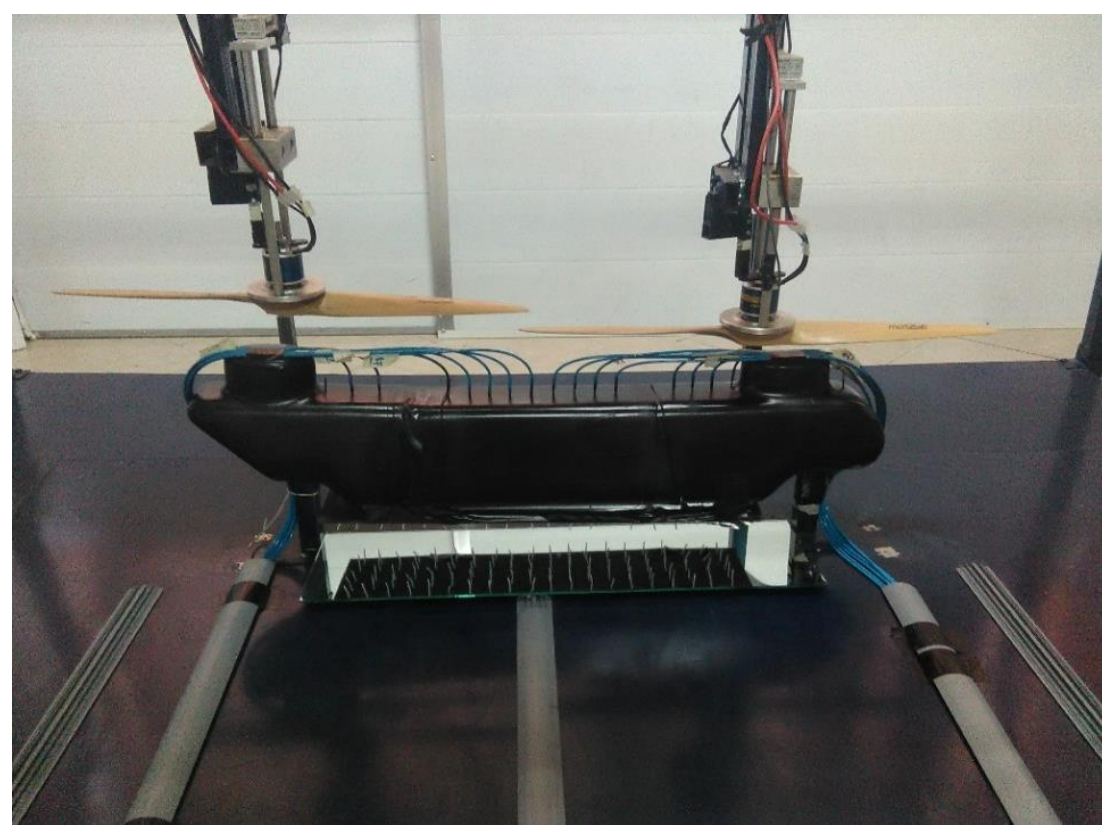

Fig. 4 White tufts on bottom surface of the model body

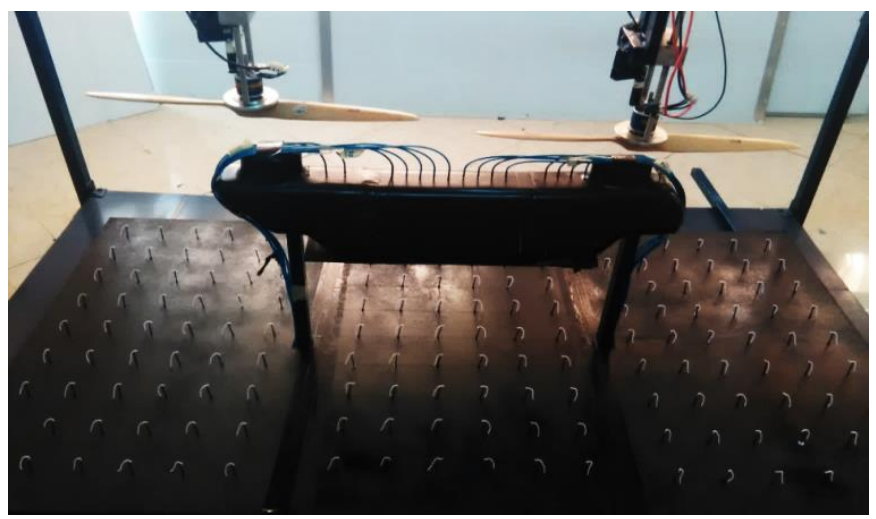

Fig. 5 White tuft flags planted at plane on the ground 


\section{Results and Discussion}

The tests and results in this study have three main subjects: the impacting fountain flow pressure and velocity variations in different points below the airframe, the influence of the twin-rotor aerodynamic on impacting fountain flow, and the flow visualization by Tuft Technique.

\subsection{The pressure and velocity under the airframe}

Fig. 6 shows the flow pressure values under the model, while only the forward rotor was rotating which simulates the baseline single-rotor configuration and protects the rotor performance from the induced flow of the other one. The measurements were performed for the forward single rotor rotational speed of $1000 \mathrm{rpm}$ and $2000 \mathrm{rpm}$. The time-dependent pressure measurements have been performed for several revolution cycles and the mean values have been presented in this paper.

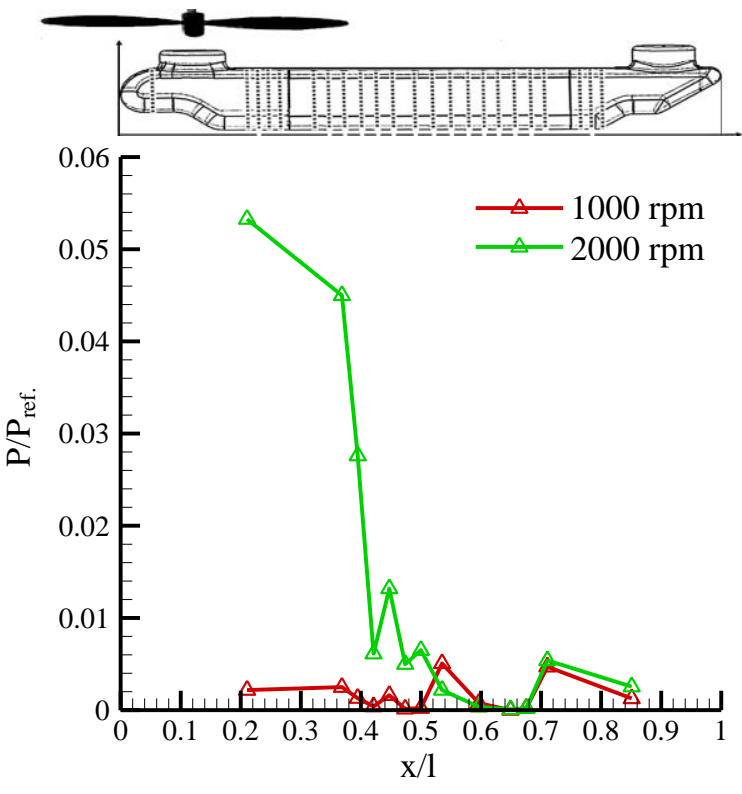

(a) $\mathrm{z} / 2 \mathrm{R}=0.5$

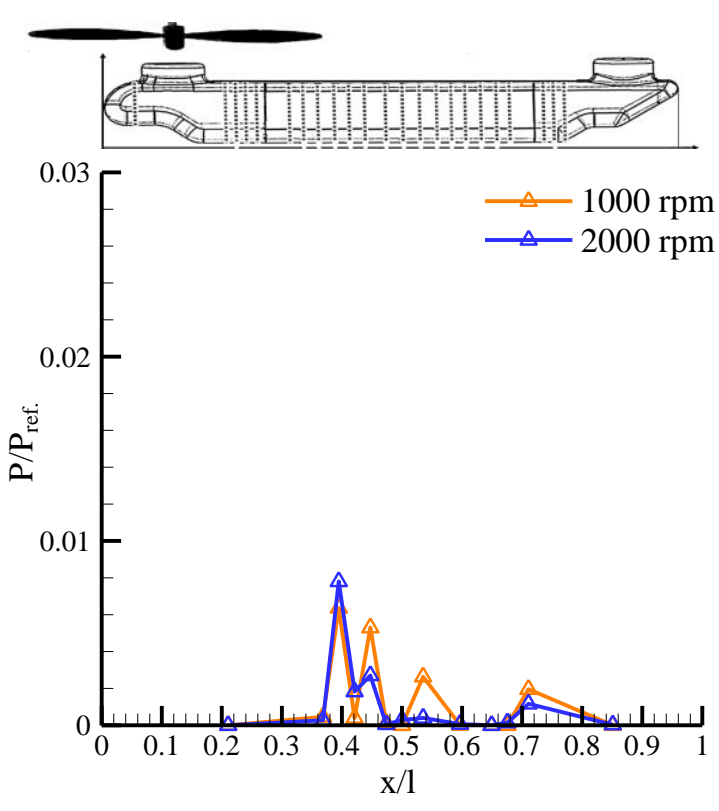

(b) $\mathrm{z} / 2 \mathrm{R}=1$

Fig. 6 pressure distribution of the Impact flow below the fuselage in single-rotor operation mode

For the single-rotor of the elevation $\mathrm{z} / 2 \mathrm{R}=0.5$, the rotational speed was equal to $1000 \mathrm{rpm}$, and the maximum pressure was recorded in the middle region of the body (Fig. 6(a)). As the rotational speed of the single-rotor was increased to $2000 \mathrm{rpm}$, the pressure in the points below the rotor increased rapidly. But unlike the previous one, in this situation maximum pressure values were occurred near the points below the mid-span of the blade. In the areas far from the middle region of the body, the increase in rotor rotational speed had little effect on pressure by moving away from the area below the rotor. Due to viscous effects, the blade tip vortices depreciate, then dissipate, and become wall jet flow. Thus, the impact flow to the body would reduce. According to the pattern of the fountain flow in the ground effect shown in Fig. 1, the flow caused by the forward blade downwash impacts with the front half of the body and leads to an increase in pressure values in these areas. In Fig. 6(b), when the elevation of the rotor increased to $\mathrm{z} / 2 \mathrm{R}=1$, the pressure distribution caused by the impact flow decreased, and rotational speed of the rotor changes had almost any significant influence on pressure distribution. This can be explained by the decrease in the ground effect. When the body and rotor elevation increased, the blocked flow which was caused by the ground surface reduced, so the impact flow reduced. It was also observed that the maximum values of the pressure were recorded in the regions below the blade tip and near the middle of the body. This was due to the interaction of the blade wake vortices with the ground and formation of the impact flow in these areas. In both cases, the impact flow pressures have been slightly reduced as moving away enough from the rotor. 


\subsection{Influence of the twin-rotor operation on impacting fountain flow}

The average values of the impacting fountain flow pressure for twin-rotor configuration are shown in Fig. 7. Due to the flow field caused by the rear rotor and the combinational effects of the twin rotors on the induced flow field, some differences can be observed in twin-rotor configuration impacting fountain flow behaviour in comparison with the single-rotor baseline.

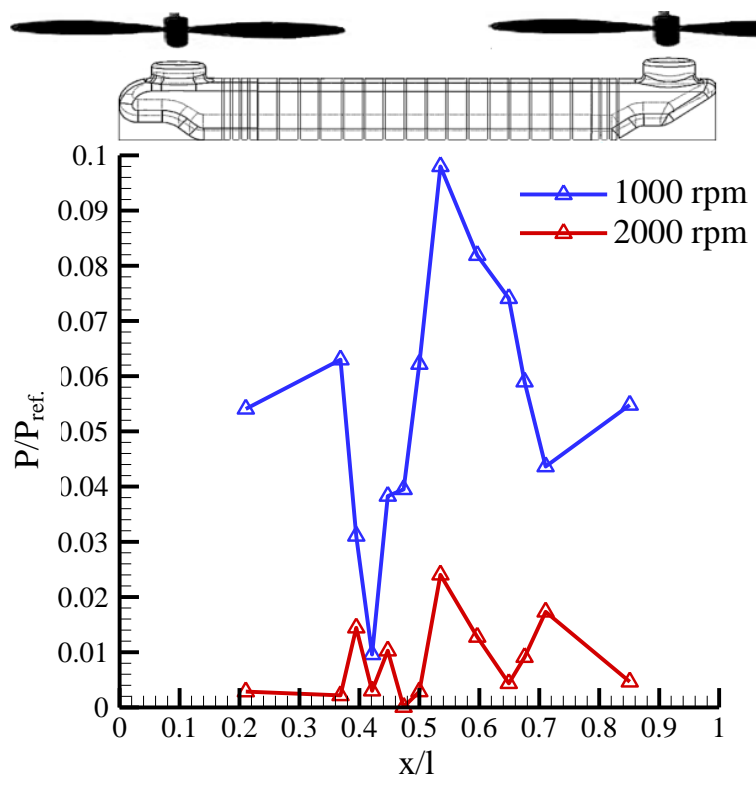

(a) $\mathrm{rpm}=1000$
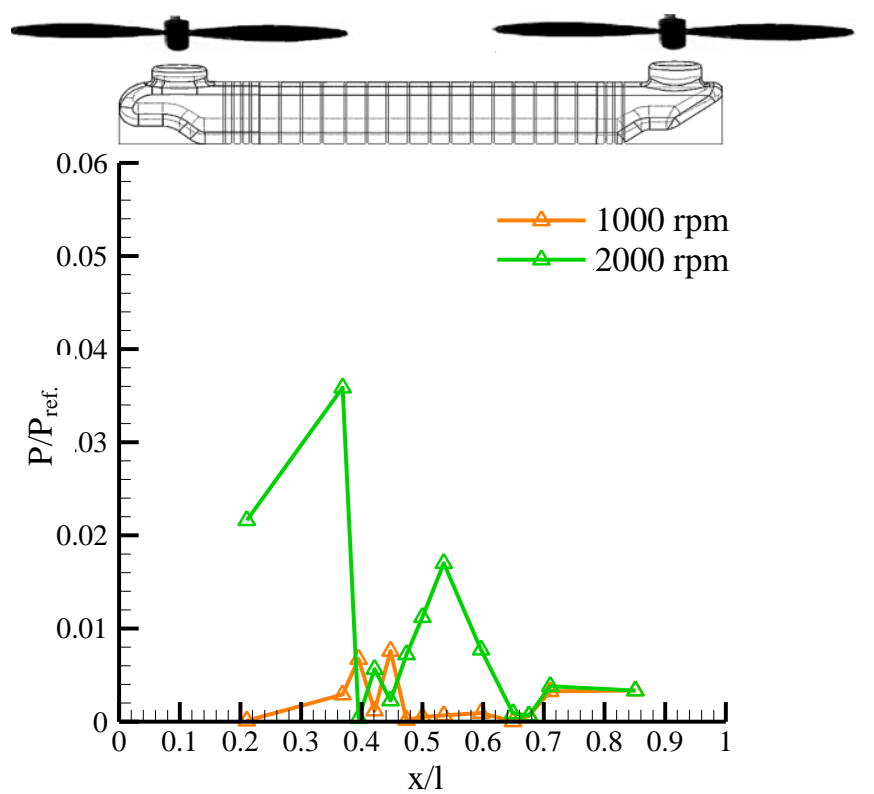

(b) $\mathrm{rpm}=2000$

Fig. 7 pressure distribution of the twin-rotor configuration Impacting fountain flow below the body

It was observed that in the twin-rotor operation, the pressure values increased more due to the impacting fountain flow. It was especially more significant at low elevation when $\mathrm{z} / 2 \mathrm{R}=0.5$ (Fig. 7(a)). The highlights in Fig. 7 are as the following. At first, as expected, increasing the rotational speed of the rotors had a significant effect on the pressure values of the impacting flow. Then, the pressure values increased by decreasing the elevation of the model. However, it should be noted that the location of the maximum pressure under the airframe changed by varying the model elevation. When $\mathrm{z} / 2 \mathrm{R}=0.5$ (Fig. 7(a)), the maximum pressure is at $\mathrm{x} / \mathrm{l}=0.57$, and when $\mathrm{z} / 2 \mathrm{R}=1$ (Fig. $7(\mathrm{~b})$ ), the maximum pressure is at $\mathrm{x} / \mathrm{l}=0.45$. In both cases, the pressure was low in the region between the $\mathrm{x} / \mathrm{l}=0.35$ and $\mathrm{x} / \mathrm{l}=0.45$. As seen in Fig. 1 , when the elevation of the model decreases, a large part of the wakes of the rotors span-wise flows recirculate. As they interact with the ground surface and meet centre line below the fuselage, it causes an upward pressure on the model. As expected, when the elevation of the model increases, the amount of blocked flow from the ground impacting to the bottom of the airframe decreases. On the other hand, owing to the decrease in the ground effects, the amount of pairing tip vortices also decreases, and the coherence of both blocked flows from the ground changes. Therefore, the location of maximum pressure varies with respect to the previous elevation.

Impacting fountain flow average velocities below the fuselage for twin-rotor configuration are shown in Fig. 8 and Fig. 9. It can be seen that in each of these graphs, there is an area where the mean velocity in bottom of the airframe is very low, and it can be said that the semi-quiescent flows were recorded in these areas. When the rotor elevation is $\mathrm{z} / 2 \mathrm{R}=0.5$, semi-quiescent flow spatial extents are the same in the both rotational speeds. But when the rotational speed of the rotors increases, the semi-quiescent flow region shifts to ahead of the model's longitudinal centre (Fig. 8).

When the model height was increased to $\mathrm{z} / 2 \mathrm{R}=1$ at $\mathrm{rpm}=1000$, the spatial extent of the semi-quiescent flow increased. The cause of this event can be explained by the fact that when the model height was increased, the amount of blocked flow from the ground surface decreased due to the decrease in the ground effect. Therefore, the flow rate decreased in the area between the rotors. When at the same elevation, the rotational speed was increased to $2000 \mathrm{rpm}$, due to the increase in the number of blade tip vortices, recirculated flow from the ground surface increased, and the spatial extent of the semi-quiescent flow decreased. 


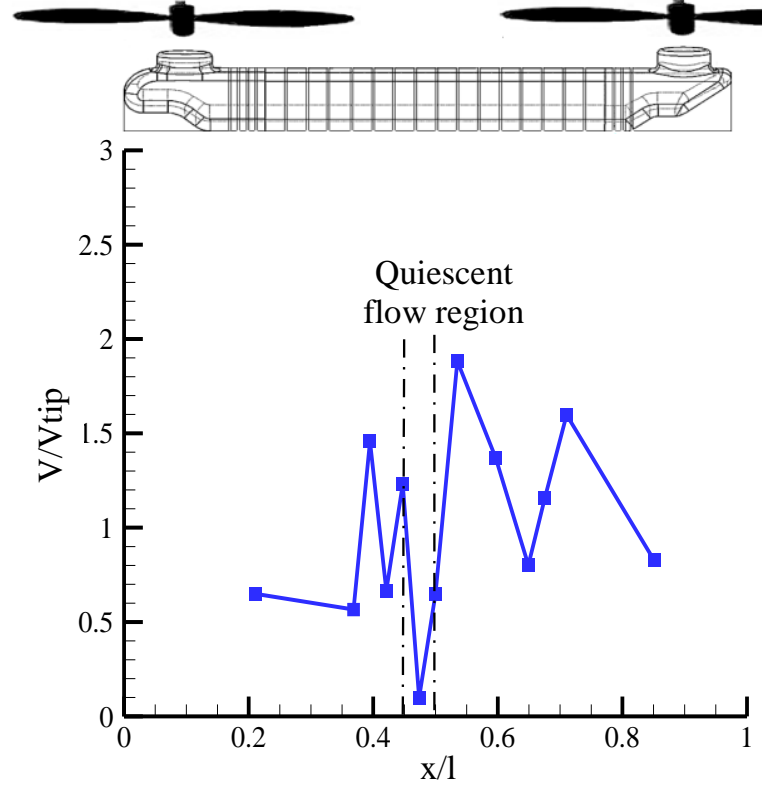

(a) $\mathrm{rpm}=1000$

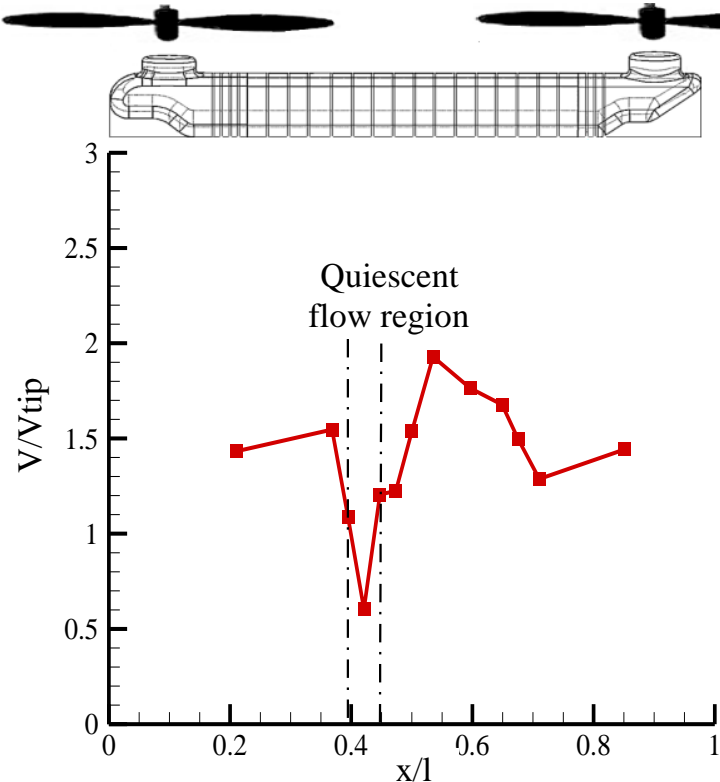

(b) $\mathrm{rpm}=2000$

Fig. 8 twin-rotor configuration Impacting fountain flow average velocity distribution below the body when $\mathrm{z} / 2 \mathrm{R}=0.5$

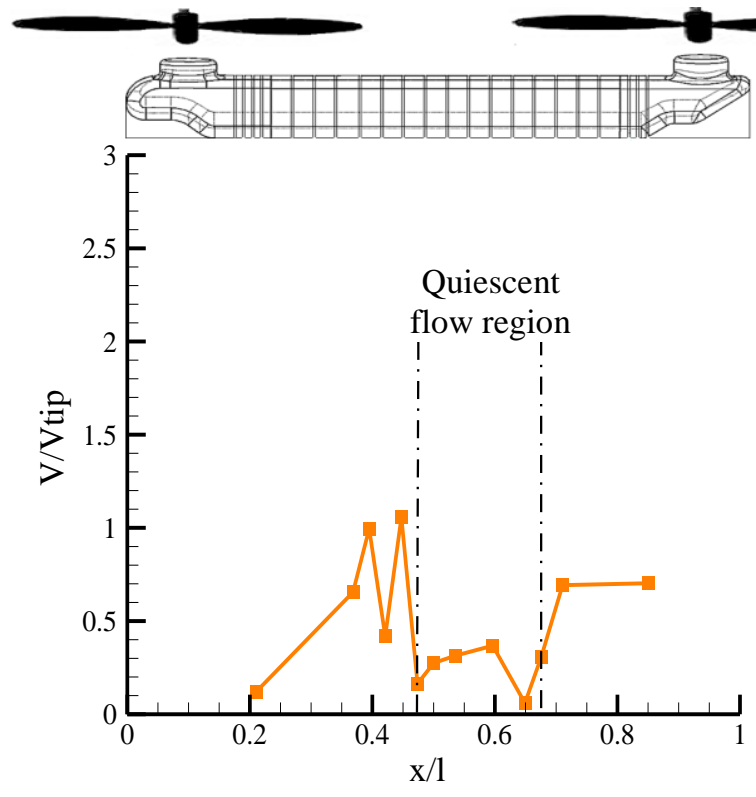

(a) $\mathrm{rpm}=1000$

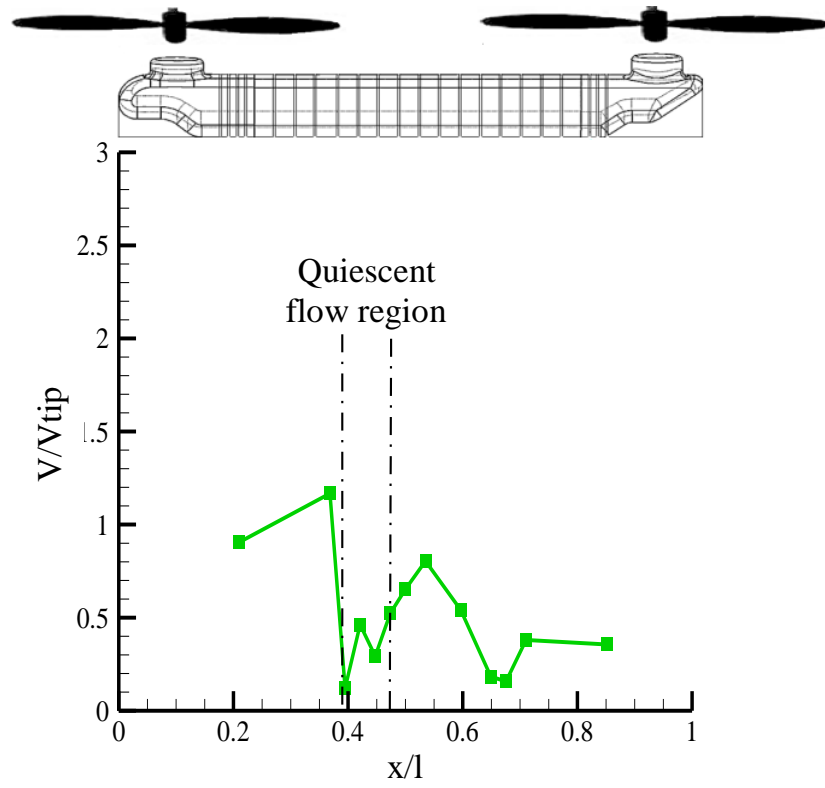

(b) $\mathrm{rpm}=2000$

Fig. 9 twin-rotor configuration Impacting fountain flow average velocity distribution below the body when $\mathrm{z} / 2 \mathrm{R}=1$

\subsection{Flow visualization}

Tuft flow visualization technique was used in the present study in order to physically understand the airflow patterns leading to the semi-quiescent and impacting flow. The tufts were extremely light and flexible, so they would move freely with the flow field. We made use of taking photos, recording the special operation videos with digital cameras, and a mirror on the ground to view the flow patterns on the bottom surface of the body (Fig. 4). Furthermore, tuft experiments were conducted for a single-rotor and a twin-rotor configuration in two elevations $(\mathrm{z} / 2 \mathrm{R}=0.5$ and $\mathrm{z} / 2 \mathrm{R}=1)$. To simulate the single-rotor configuration which was isolated from induced flow of the other one, tuft flow visualization was conducted in elevation $\mathrm{z} / 2 \mathrm{R}=0.5$ for that (Fig. 10).

It can be seen from the flow pattern in Fig. 10, when the single-rotor was operating, a large part of the flow at the bottom of the model body was lengthwise flowing toward its tail. When a twin-rotor configuration hovers near the ground, the 
direction of the induced flow by the rotor changes from vertical downwash to radial outwash because of interactions with ground. Therefore, the outwash starts and flows away from the model between the ground and bottom surface. Since the front rotor was working in this experiment, the outwash flow started at the bottom surface behind the centre of this rotor and moved toward the tail.
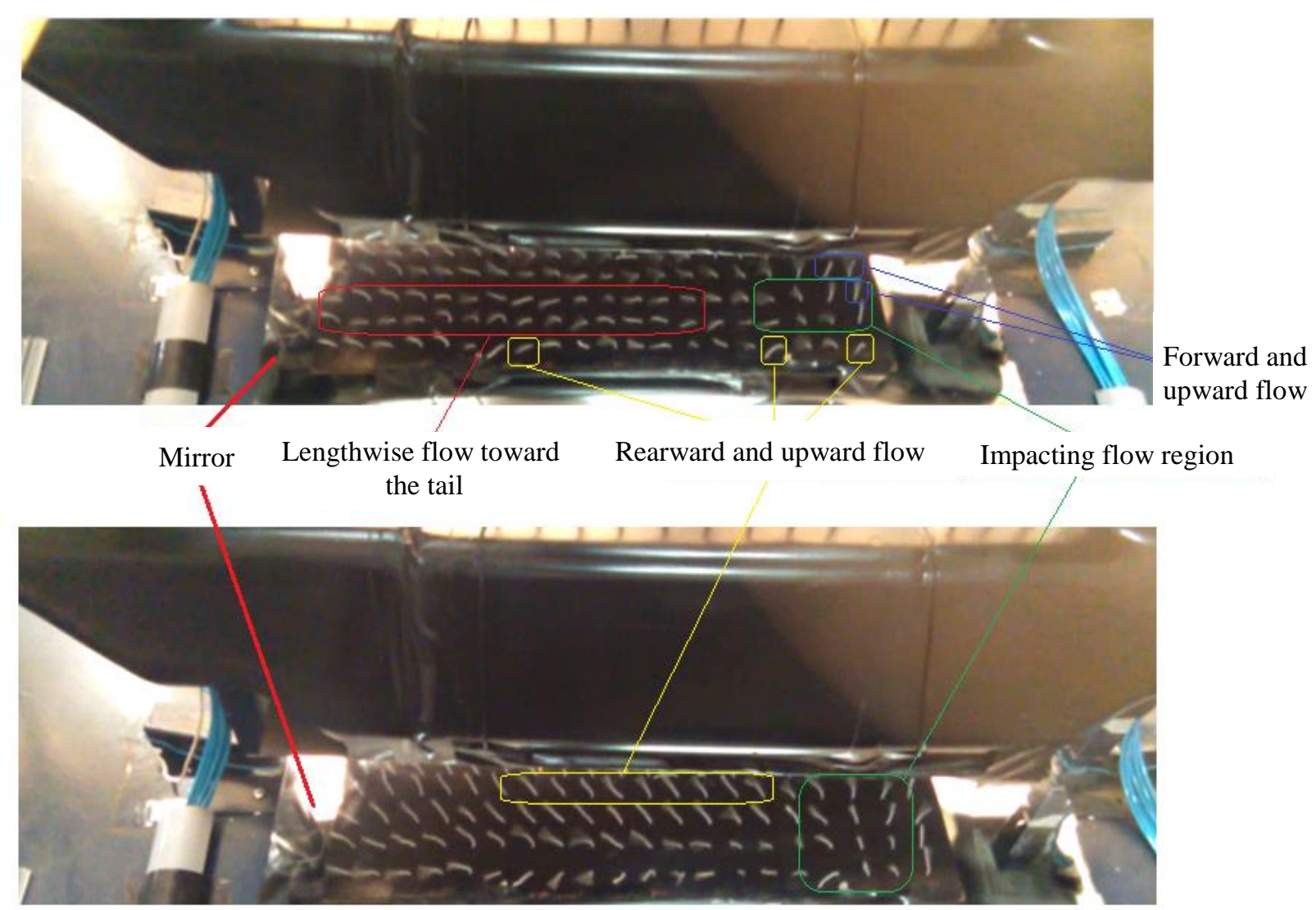

Fig. 10 Tuft flow visualization of bottom surface in single-rotor operation mode in rpm=2000

At some points on the edges of the bottom surface, there were rearward and upward flows. These flows were on the side-walls of the airframe due to the pressure difference below and above the body. However, owing to the shape of the body and the adverse pressure gradient in the upstream parts of the side walls, upward flow may be separated from the side-walls surfaces. The flow separation on the walls creates an almost vacuum area in the upstream areas, so it creates a positive effect on the overall lift. In the front underneath the body, there was an impinging jet-like small region. As shown in the Fig. 6(a), in this area that located between $\mathrm{x} / \mathrm{l}=0.2$ to 0.4 , the pressure value is maximum. It is understood that there is an area that rotor induced flow is subdivided into forward outwash, backward outwash, and upward side flows there.

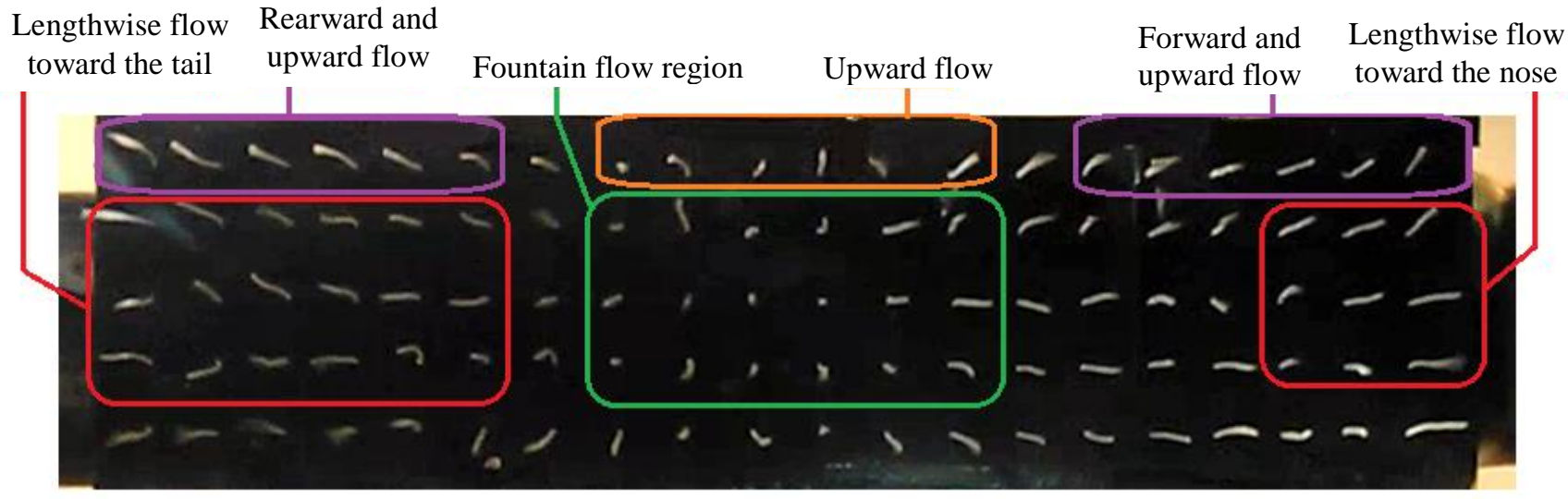

Fig. 11 Tuft flow visualization of bottom surface for twin-rotor operation mode in rpm=2000 


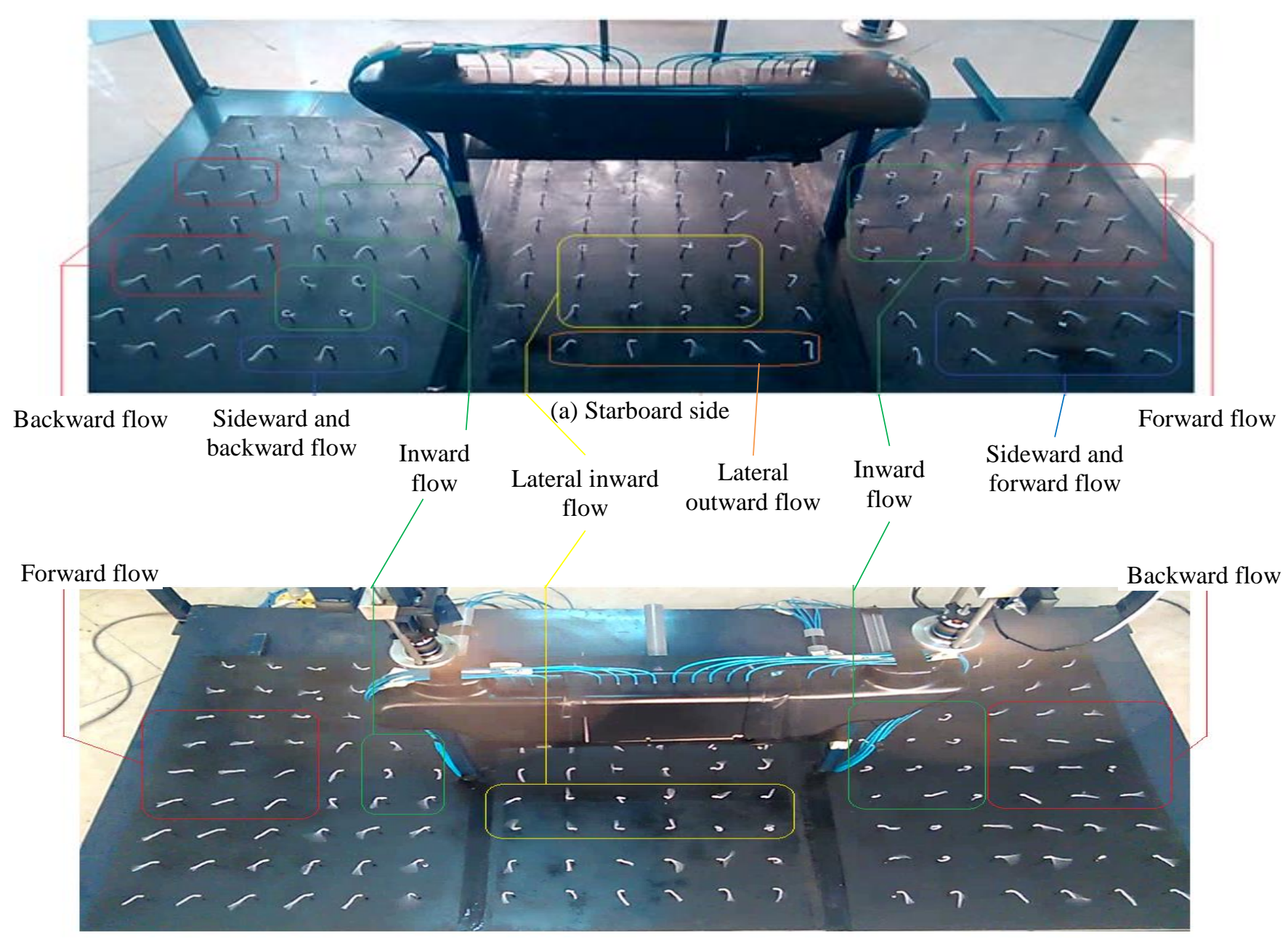

(b) Port side

Fig. 12 Tuft flow visualization on the ground for twin-rotor configuration

Fountain flow tuft flow visualization tests which were conducted for the twin-rotor configurations showed that there are some fundamental differences in the behaviour of this configuration comparing to the single-rotor baseline, as a result of combinational effects of the twin rotors on the induced flow field. In Fig. 11, it is seen that the fountain flow region was located in the longitudinal central area at the bottom of the body ( $\mathrm{x} / \mathrm{l}=0.45$ to 0.6 ). It can also be obtained in Fig. 7(b) that the maximum pressure occurs at $\mathrm{x} / \mathrm{l}=0.57$ when $\mathrm{z} / 2 \mathrm{R}=1$. So, tuft flow visualization experiments confirm the accuracy of the measurements. At an elevation of $z / 2 R=0.5$, the impact flow on the bottom of the body was extremely disturbed due to the longer life of the rotor wake vortices in the wall jet and their oscillatory nature. Consequently, the movements and orientations of the tufts are very unstable and fluctuating, so the flow ranges, especially the impacting fountain flow range, are constantly changed, and detecting the flow pattern is also very difficult. In this case, like single-rotor operation, there are forward, rearward, and upward flows. The cause of such flows is due to the effects of the fountain flow and have positive effects on the lifting force and the aerodynamic performance of the rotors.

In Fig. 12, it is seen that there are longitudinal and lateral inward airflow regions below the nose and tail and between the rotors respectively. Tuft flow visualization experiments showed these phenomena at both altitudes for such configurations. The cause of such flows was the effects of the upward fountain flow between the twin-rotor which creates a suction on the central flow.

\subsection{Thrust measurements}

To measure the effect of the fountain flow on the efficiency of twin-rotor configurations in hover flight, the values of the thrusts produced in the ground effects and out of ground effect are measured in hover and are shown in in Fig. 13. 
Between the tandem rotors, increase in sub-body pressure values because the impacting flow creates a resultant lift force beneath the body. This upward force was added to the thrust values. Therefore, it was observed that the ground proximity had more positive effects on twin-rotor efficiency than single-rotor. To validate the results, the measured thrust values in the present study were compared with the results obtained from the flight tests of several types of single-rotor helicopters .The results of these comparisons confirm the positive effects of fountain flow on the performance of twin-rotor configurations.

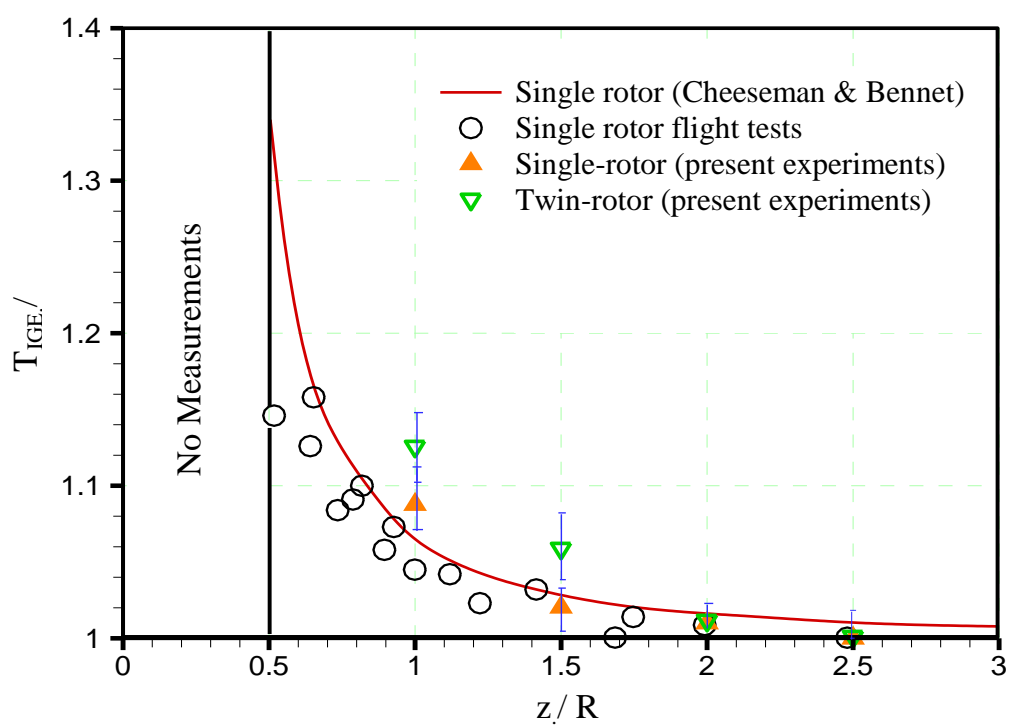

Fig. 13 Thrust values of the single and twin rotors

\subsection{Uncertainty analysis}

To measure the uncertainty in this study, some tests were repeated five times. The uncertainty of the results is the product of the standard to a values called t-distribution, which are expressed in Table 1 [19], depending on the number of observations and considered confidence intervals. By calculating the mean and standard deviation of the pressure values which was measured by ports and the thrust values, and took into account the $95 \%$ confidence interval, the mean uncertainty at the present results was calculated $5.6 \%$. In other words, if the experiments were repeated under the same conditions, probably the $95 \%$ of new results would be on average 5.6\% different from the results presented in this paper. Factors such as noises caused by sensors working together, error in data transfer terminals, frictional loss due to pneumatic tubes lengths, and oscillations caused by rotors are evaluated as factors in the uncertainty of the results. Fig. 13 shows the uncertainty in the measurement of thrust values as an example.

Table 1 t-Distribution values for 5 degrees of freedom

\begin{tabular}{|c|c|c|c|c|c|c|c|c|c|c|c|}
\hline One-sided & $75 \%$ & $80 \%$ & $85 \%$ & $90 \%$ & $95 \%$ & $97.5 \%$ & $99 \%$ & $99.5 \%$ & $99.75 \%$ & $99.9 \%$ & $99.95 \%$ \\
\hline Two-sided & $50 \%$ & $60 \%$ & $70 \%$ & $80 \%$ & $90 \%$ & $95 \%$ & $98 \%$ & $99 \%$ & $99.5 \%$ & $99.8 \%$ & $99.9 \%$ \\
\hline 1 & 1.000 & 1.376 & 1.963 & 3.078 & 6.314 & 12.71 & 31.82 & 63.66 & 127.3 & 318.3 & 636.6 \\
\hline 2 & 0.816 & 1.080 & 1.386 & 1.886 & 2.920 & 4.303 & 6.965 & 9.925 & 14.09 & 22.33 & 31.60 \\
\hline 3 & 0.765 & 0.978 & 1.250 & 1.638 & 2.353 & 3.182 & 4.541 & 5.841 & 7.453 & 10.21 & 12.92 \\
\hline 4 & 0.741 & 0.941 & 1.190 & 1.533 & 2.132 & 2.776 & 3.747 & 4.604 & 5.598 & 7.173 & 8.610 \\
\hline 5 & 0.727 & 0.920 & 1.156 & 1.476 & 2.015 & 2.571 & 3.365 & 4.032 & 4.773 & 5.893 & 6.869 \\
\hline
\end{tabular}

\section{Conclusions}

A series of the tests have been performed by using a multipurpose test stand to study the impacting fountain flow pattern for both single and twin-rotor configurations at two elevations from the ground surface. The pressure and velocity measurements were performed by the pressure ports drilled longitudinally along the fuselage. The rotors and the fuselage have been scaled from a generic twin-rotor configuration airframe. 
(1) For a single-rotor, the results have shown that the maximum values of the pressure were recorded in regions below the blade tip and near the middle of the body.

(2) Tuft flow visualization tests conducted for twin-rotor configurations showed that there were some fundamental differences in flow patterns below them which caused by impacting flow formation comparing to the single-rotor baseline.

(3) Pressure measurements and tuft flow visualization under the twin-rotor configuration body showed that an impacting fountain flow was between the rotors in the central region below the body.

(4) The results showed that when twin-rotor configuration hovered near the ground, the pressure distribution was greatly increased due to the impacting fountain flow, especially this increase was more significant when decreasing the height of model. So, the ground effects improves the pressure values of impacting flow and thrust more than single-rotor configuration.

(5) In general, it can be said that the presence of the fountain flow in twin-rotor configuration has positive effects such as increasing the lifting force and the pressure distribution balance. These aerodynamic effects must be taken into account in the design of the flight controls trims and stability systems of twin-rotor configurations.

\section{Conflicts of Interest}

The authors declare no conflict of interest.

\section{References}

[1] M. Silva and R. Riser, "CH-47D tandem rotor outwash survey," Proc. of American Helicopter Society 67th Annual Forum, vol. 4, December 2007, pp. 57-64.

[2] G. J. Leishman, Principles of helicopter aerodynamics with CD extra, Cambridge, New York, Melbourne, Madrid, Cape Town, Singapore , 2006.

[3] F. F. Felker, J. S. Light, “Aerodynamic interactions between a rotor and wing in hover," Journal of the American Helicopter Society, vol. 33, no. 2, pp. 53-61, April 1988.

[4] M. Mcveigh, "The V-22 tilt-rotor large-scale rotor performance/wing download test and comparison with theory," Vertica, vol. 10, no. 3, pp. 281-297, January 1986.

[5] D. R. Polak, W. Rehm, and A. R. George, "Effects of an image plane on the tilt rotor fountain flow," Journal of the American Helicopter Society, vol. 45, no. 2, pp. 90-96, April 2000.

[6] M. Ramasamy, M. Potsdam, and G. K. Yamauchi, "Measurements to understand the flow mechanisms contributing to tandem-rotor outwash," 71st Annual Forum and Technology Display, Virginia Beach, vol. 1, pp. 612-647, January 2015.

[7] V. Gupta and J. D. Baeder, "Quad tilt rotor aerodynamics in helicopter mode," Annual Forum Proceedings-American Helicopter Society, vol. 61, no. 1, p. 416, January 2005.

[8] M. Ramasamy and J. G. Leishman, "Interdependence of diffusion and straining of helicopter blade tip vortices," Journal of Aircraft, vol. 41, no. 5, pp. 1014-1024, September-October 2004.

[9] B. Johnson, J. G. Leishman, and A. Sydney, "Investigation of sediment entrainment using dual-phase, high-speed particle image velocimetry," Journal of the American Helicopter Society, vol. 55, no. 4, pp. 42003-42003, October 2010.

[10] J. Wayne, Helicopter Theory, Princeton: Princeton University Press, 1980.

[11] W. Stepniewski, "A simplified approach to the aerodynamic rotor interference of tandem helicopters," Proceedings of Annul Western Forum, vol. 2, pp. 71-90, September 1955.

[12] F. F. Felker and J. S. Light, "Aerodynamic interactions between a rotor and wing in hover," Journal of the American Helicopter Society, vol. 33, no. 2, pp. 53-61, July 1986.

[13] Y. You, D. Na, and S. N. Jung, "Improved rotor aeromechanics predictions using a fluid structure interaction approach," Aerospace Science and Technology, vol. 73, pp. 118-128, December 2017.

[14] A. F. Antoniadis, D. Drikakis, B. Zhong, G. Barakos, R. Steijl, and M. Biava et al., "Assessment of CFD methods against experimental flow measurements for helicopter flows," Aerospace Science and Technology, vol. 19, no.1, pp. 86-100, June 2012. 
[15] G. M. Eberhart, "Modeling of ground effect benefits for multi-rotor small unmanned aerial systems at hover," Master of Science (MS), Dept. Engineering and Technology, Ohio University, 2017.

[16] L. Ye, Y. Zhang, S. Yang, X. Zhu, and J. Dong, "Numerical simulation of aerodynamic interaction for a tilt rotor aircraft in helicopter mode," Chinese Journal of Aeronautics, vol. 29, no.4, pp. 843-854, August 2016.

[17] J. F. Tan, T. Y. Zhou, Y. M. Sun, and G. N. Barakos, "Numerical investigation of the aerodynamic interaction between a tiltrotor and a tandem rotor during shipboard operations," Aerospace Science and Technology, vol. 87, pp. 62-72, February 2019.

[18] Q. Wang and Q. Zhao, "Rotor aerodynamic shape design for improving performance of an unmanned helicopter," Aerospace Science and Technology, vol. 87, pp. 478-487, April 2019.

[19] H. Pham, Springer handbook of engineering statistics, Springer Science \& Business Media, 2006.

Copyright $\subseteq$ by the authors. Licensee TAETI, Taiwan. This article is an open access article distributed under the terms and conditions of the Creative Commons Attribution (CC BY-NC) license (https://creativecommons.org/licenses/by-nc/4.0/). 